\title{
Food Insecurity and Depressive Symptoms in Adolescents Aged 12-15 Years from Low- and Middle-Income Countries
}

\author{
Meng Wang* \\ School of Sports and Health, Nanjing Sport of Institute, Nanjing, 210014, China \\ ${ }^{*}$ Corresponding Author: Meng Wang. Email: njtywm@163.com \\ Received: 27 February 2021 Accepted: 04 April 2021
}

\begin{abstract}
Purpose: Little is known about the role of food insecurity (FIS) on depressive symptoms among adolescents. Thus, this study aimed to explore the association between FIS and depressive symptoms among adolescents aged 12-15 years from low- and middle-income countries across the world. Methods: Data from the Global schoolbased Student Health Survey were analyzed in 51,702 adolescents [mean (SD) age 13.8 (1.0) years; 49.3\% girls). Self-reported measures assessed depressive symptoms during the past 12 months, and food insecurity. Participants reporting yes for depressive symptoms. FIS was categorized into five levels, including 'never', 'rarely', 'sometimes', 'most of the time' and 'always'. Multivariable logistic regression analysis was performed, and a country-wise meta-analysis was undertaken to compare country difference in the associations between FIS and depressive symptoms. Results: The prevalence of depressive symptoms was $30.0 \%$, respectively. Compared with those reporting never for FIS, adolescents with increased severity of FIS were more likely to report depressive symptoms regardless of gender. Country-wise meta-analysis demonstrated that having FIS versus not having FIS was associated with $60 \%$ greater odds for depressive symptoms ( $\mathrm{OR}=1.60$; $95 \% \mathrm{CI}: 1.52-1.69)$ but with a moderate between-country heterogeneity $\left(I^{2}=12.7 \%\right)$. Conclusion: The current study indicates that alleviating FIS may be an effective prevention against depressive symptoms among adolescents from LMICs. Future studies should adopt improved study design to confirm or negate our research findings, which informs more efficient public mental health interventions.
\end{abstract}

\section{KEYWORDS}

Food insecurity; depressive symptoms; global school-based health survey; low- and middle-income countries

\section{Introduction}

Depression refers to low mood and anhedonia as the core symptoms, with abnormal weight gain and loss, insomnia or drowsiness as additional symptoms a mood dysregulation [1]. Globally, more than 264 million people of all ages suffer from depressive symptoms, which has caused a serious social and economic burden [2]. It is predicted that by 2030, depression will has become the first leading cause of the global burden of disease factors [3].

Adolescence is not only a period of the first appearance and rapid development of individual depressive symptoms [4,5], but also a period of high incidence and prevalence of depressive symptoms [6], which is more likely to link with later severe depressive disorders. With this regard, an increasing number of 
studies focusing on depression in adolescents has been conducted for exploring effective approaches to against this mental health disorder [7,8]. Recently, two meta-analysis studies have found that the prevalence of depressive symptoms in adolescents ranged between $5.3 \%-29.2 \%$ and $2.2 \%-20 \%[9,10]$. The higher prevalence of depressive symptoms is liked with adverse health outcomes, like suicidality [11], serious social and cognitive impairments [12], school dropout [13], smoking and alcohol abuse [14]. Therefore, adolescent' depressive symptoms is an urgent health problem that should be prevented.

To better understand adolescents' depressive symptoms, it is necessary to determine its correlates and determinants, which is conducive to design and implement effective mental health interventions. There are various correlates of depressive symptoms in adolescents [15-19]. Food insecurity (FIS) can be defined as the inability to obtain sufficient or appropriate food because of financial constraints [20]. According to the previous studies, food insecurity was associated with poorer physical health (e.g., headache, stomachache, difficulty of breathing [21]) and mental health (e.g., depression and anxiety [22], non-cognitive performance [23], suicide ideation [24]). However, little is known about the association between food insecurity and depressive symptoms in adolescents, to the date and author's knowledge. Compared with it, in adults, much evidence has shown that FIS was an important of depressive symptoms. For example, data from 2015 Korean Community Health Survey revealed the association between FIS and experience of depressive symptoms in people aged over 18 years [25]. Among Mexican population, there was evidence on the association between FIS and depressive symptoms [26]. A 2018 review focusing on females from high income countries concluded that FIS may contribute to depressive symptoms [27]. In a review study with meta-analysis, the authors found that FIS was positively associated with higher risks of depression $(\mathrm{OR}=1.40 ; 95 \% \mathrm{CI}: 1.30-1.58)$ [28]. These evidences may inform researchers to hypothesize that FIS associates with depressive symptoms in adolescents. Owing to insufficient evidence based on adolescents, it is needed to conduct more studies in this population.

In addition to the above-mentioned research gaps, there are other considerations for this study. It is wellrecognized that adolescents from low- and middle-income countries (LMICs) are highly likely to be exposed to FIS, which in turn make these adolescents subject to health burden, including mental and emotional health risks [29-31]. However, previous studies paid less attention on those from LMICs. To address the aforementioned research gaps in the literature, this study aimed to explore the associations between FIS and depressive symptoms in adolescents from LMICs.

\section{Methods}

\subsection{Study Survey}

Publicly available data from the Global school-based Student Health Survey (GSHS) were analyzed. Details on this survey can be found at http://www.who.int/chp/gshs and http://www.cdc.gov/gshs. Briefly, the GSHS was jointly developed by the World Health Organization and the United States Centers for Disease Control and Prevention (its associated the Youth Risk Behavior Survey, YRBS) and other United Nations allies. The core aim of the survey was to assess and quantify risk and protective factors of major non-communicable diseases. The survey used a standardized two-stage probability sampling design for the selection process within each participating country. For the first stage, schools were selected with probability proportional to size sampling. The second stage involved the random selection of classrooms which included students within each selected school. All students in the selected classrooms were eligible to participate in the survey regardless of age.

Data collection was performed during one regular class period. The questionnaire was translated into the local language in each country and consisted of multiple-choice response options. Students recorded their response on computer scanned sheets. All GSHS surveys were approved, in each country, by both a national government administration (most often the Ministry of Health or Education) and an institutional review board or ethics committee. Student privacy was protected through anonymous and voluntary 
participation, and informed consent was obtained as appropriate from the students, parents, and/or school officials. Data were weighted for non-response and probability selection. Country survey having variables of active school travel and depressive symptoms were included into this study. Because the GSHS is an iterative survey study with updates every 4 or 5 years, the latest dataset of each survey was included in the present analysis (year: 2003-2008).

\subsection{Food Insecurity (Exposure)}

Food insecurity was assessed by the question "During the past 30 days, how often did you go hungry because there was not enough food in your home?" The answers consisted of always, most of the time, sometimes, rarely, and never. This item was used in the previous studies [19,32,33]. In the present study, we classify variable of food insecurity into two groups, yes (always, most of the time, sometimes) vs. no (rarely and never) where some parts of analysis are needed and appropriate. There is evidence to support the question's reliability and validity based on the Youth Risk Behavior Survey (https://www.cdc.gov/ $\mathrm{mmwr} / \mathrm{pdf} / \mathrm{rr} / \mathrm{rr6201}$.pdf), which shows acceptable validity and reliability.

\subsection{Depressive Symptom (Outcome)}

Depressive symptoms were assessed by one single question "During the past 12 months, did you ever feel so sad or hopeless almost every day for two weeks or more in a row that you stopped doing your usual activities?" Responses were yes and no. Participants answering yes were considered as having depressive symptoms, in line with previous studies [19,34,35]. There is evidence to support the question's reliability and validity based on the Youth Risk Behavior Survey (https://www.cdc.gov/mmwr/pdf/rr/rr6201.pdf), which shows acceptable validity and reliability.

\subsection{Control Variables}

Gender, age, physical activity and sedentary behavior were regarded as covariates based on previously published research [19,32-35]. To assess levels of physical activity, a reliable and valid measure was used. The questions asked about the number of days with physical activity of at least 60 min during the past 7 days. Sedentary behavior was assessed with the question "How much time do you spend during a typical or usual day sitting and watching television, playing computer games, talking with friends, or doing other sitting activities?" with answer options: $<1,1-2,3-4,5-6,7-8$, and $\geq 8 \mathrm{~h} /$ day.

\subsection{Statistical Analysis}

All the statistical analyses were performed with STATA 16.1. For descriptive analyses, the prevalence of FIS and depressive symptoms was estimated, respectively. The prevalence of FIS and depressive symptoms by gender and country was also calculated. Multivariable logistic regression analysis was implemented to assess the association between FIS and depressive symptoms (overall, by gender and country). The regression was analysis was adjusted for age, gender, physical activity, sedentary behavior, as well as country, with the exception of the gender-stratified and country-wise analyses which were not adjusted for gender and country, respectively.

Random effects meta-analysis was used to generate pooled estimates of the association between FIS and depressive symptoms for the overall sample and sex-stratified sample, using DerSimonian and Laird method. To assess the level of between-country heterogeneity, the Higgin's $I^{2}$ statistic was calculated based on country-wise estimates. This represents the degree of heterogeneity that is not explained by sampling error with a value of $<40 \%$ often considered as negligible and $40 \%-60 \%$ as moderate heterogeneity. A pooled estimate was obtained by combining the estimates for each country into a random effect metaanalysis. All variables were included in the regression analyses as categorical variables with the exception of age and PA (continuous variables). Taylor linearization methods were used in all analyses to account for the sample weighting and complex study design. Results from the logistic regression analyses are presented as odds ratios with $95 \%$ confidence intervals (CIs). The statistical significance level was $p<0.05$ with being two sided. 


\section{Results}

Tab. 1 shows sample characteristics and prevalence of FIS and depressive symptoms in study participants from 26 LMICs in this study. In total, 51,702 adolescents aged 12-15 years were included in the analyses. Among these adolescents, 50.1\% participants reported food insecurity at being 'never', and the prevalence of reporting being 'rarely', 'sometimes', 'most of the time' and 'always' for depressive symptoms were $21.2 \%, 22.5 \%, 4.1 \%$ and $2.1 \%$, respectively. Besides, $30.1 \%$ of adolescents reported having depressive symptoms affirmatively. The prevalence of FIS and depressive symptoms by each included country can be found at Tab. 1.

Table 1: Sample characteristics and prevalence of food insecurity and depressive symptoms in this study

\begin{tabular}{|c|c|c|c|c|c|c|c|c|c|}
\hline \multirow[t]{2}{*}{ Country } & \multirow[t]{2}{*}{$\mathrm{N}$} & \multirow[t]{2}{*}{ Year } & \multirow{2}{*}{$\begin{array}{l}\text { Response } \\
\text { rate }(\%)\end{array}$} & \multicolumn{5}{|c|}{ Food insecurity (\%) } & \multirow{2}{*}{$\begin{array}{l}\text { Depressive } \\
\text { symptoms (\%) }\end{array}$} \\
\hline & & & & Never & Rarely & Sometimes & $\begin{array}{l}\text { Most of } \\
\text { the time }\end{array}$ & Always & \\
\hline Argentina & 1403 & 2007 & 77.1 & 68.3 & 18.8 & 10.4 & 1.2 & 1.4 & 29.6 \\
\hline Botswana & 1252 & 2005 & 95 & 36.9 & 6.9 & 41.9 & 8.8 & 5.5 & 39.4 \\
\hline Chile & 1900 & 2004 & 85 & 75.2 & 17.1 & 6.0 & 1.2 & 0.5 & 30.8 \\
\hline China & 2126 & 2003 & 99 & 58.3 & 28.3 & 11.1 & 1.8 & 0.5 & 18.3 \\
\hline Djibouti & 913 & 2007 & 83 & 53.4 & 7.7 & 20.3 & 5.7 & 12.9 & 37.7 \\
\hline Ecuador & 1716 & 2007 & 85.6 & 60.4 & 29.6 & 7.2 & 1.5 & 1.3 & 25.4 \\
\hline Egypt & 4475 & 2006 & 87 & 57.9 & 22.5 & 14.5 & 3.4 & 1.7 & 35.3 \\
\hline Grenada & 1127 & 2007 & 78 & 57.5 & 13.5 & 21.8 & 5.1 & 2.2 & 23.2 \\
\hline Guyana & 980 & 2004 & 80 & 57.2 & 11.9 & 22.5 & 4.1 & 4.3 & 32.8 \\
\hline India & 6553 & 2007 & 83 & 66.5 & 16.7 & 13.2 & 2.3 & 1.4 & 24.4 \\
\hline Indonesia & 2939 & 2007 & 93.1 & 35.7 & 27.1 & 31.5 & 4.5 & 1.2 & 21 \\
\hline Jordan & 1490 & 2007 & 100 & 49.7 & 21.2 & 15.9 & 7.8 & 5.4 & 37.1 \\
\hline Kenya & 2403 & 2005 & 84 & 38.6 & 13.4 & 33.8 & 10.0 & 4.2 & 47.9 \\
\hline Morocco & 1838 & 2006 & 84 & 54.7 & 17.4 & 18.8 & 7.1 & 2.0 & 37.2 \\
\hline Philippines & 3792 & 2003 & 79 & 31.0 & 28.1 & 33.4 & 5.0 & 2.5 & 40.2 \\
\hline Seychelles & 969 & 2007 & 82 & 41.4 & 15.6 & 26.3 & 10.8 & 5.9 & 32.1 \\
\hline Sri Lanka & 2388 & 2008 & 89 & 67.1 & 13.0 & 13.2 & 2.8 & 3.9 & 31.8 \\
\hline St Lucia & 1000 & 2007 & 82 & 56.6 & 17.2 & 20.7 & 4.3 & 1.1 & 23.3 \\
\hline St Vincent & 1022 & 2007 & 84 & 59.1 & 11.0 & 23.2 & 3.8 & 3.0 & 27.5 \\
\hline Tanzania & 1635 & 2006 & 87 & 72.0 & 16.6 & 7.6 & 2.3 & 1.4 & 24 \\
\hline Thailand & 2490 & 2008 & 93 & 47.4 & 22.5 & 26.6 & 1.7 & 1.8 & 16.6 \\
\hline Tunisia & 2391 & 2008 & 83 & 59.1 & 14.9 & 18.3 & 5.1 & 2.6 & 36.1 \\
\hline Uganda & 1646 & 2003 & 69 & 48.5 & 12.8 & 30.0 & 4.5 & 4.2 & 39.7 \\
\hline Uruguay & 421 & 2006 & 71.2 & 80.9 & 11.9 & 5.3 & 0.3 & 1.6 & 17.6 \\
\hline Venezuela & 1867 & 2004 & 86 & 77.8 & 9.1 & 9.3 & 0.8 & 3.0 & 22 \\
\hline Zambia & 966 & 2004 & 70 & 18.0 & 9.9 & 42.6 & 21.7 & 7.8 & 51.4 \\
\hline Overall & 51702 & 1 & / & 50.1 & 21.2 & 22.5 & 4.1 & 2.1 & 30.1 \\
\hline
\end{tabular}

Note: The prevalence of food insecurity and depressive symptoms was weighed. 
Fig. 1 illustrates the prevalence of depressive symptoms by levels of FIS. Among adolescents, $25.8 \%$ of participants reported having depressive symptoms, and more females have depressive symptoms than males $(27.7 \%>23.6 \%)$. Among food insecure adolescents, those adolescents who are food insecure most of the time present highest percentage of having depressive symptoms compared with counterparts of other three levels of food insecurity (most of the time: $45.2 \%>$ always: $41.8 \%>$ sometimes: $35.9 \%>$ rarely: 29.9\%). More female adolescents have depressive symptoms than males in four levels of food insecurity group (most of the time: $48.5 \%>42.3 \%$; always: $45.5 \%>40.2 \%$; sometimes: $38.0 \%>33.7 \%$; rarely: $30.3 \%>29.5 \%$ ). In addition, percentages of having depressive symptoms among females are all above overall ones, while percentages of males are all below overall ones, regardless of levels of FIS.

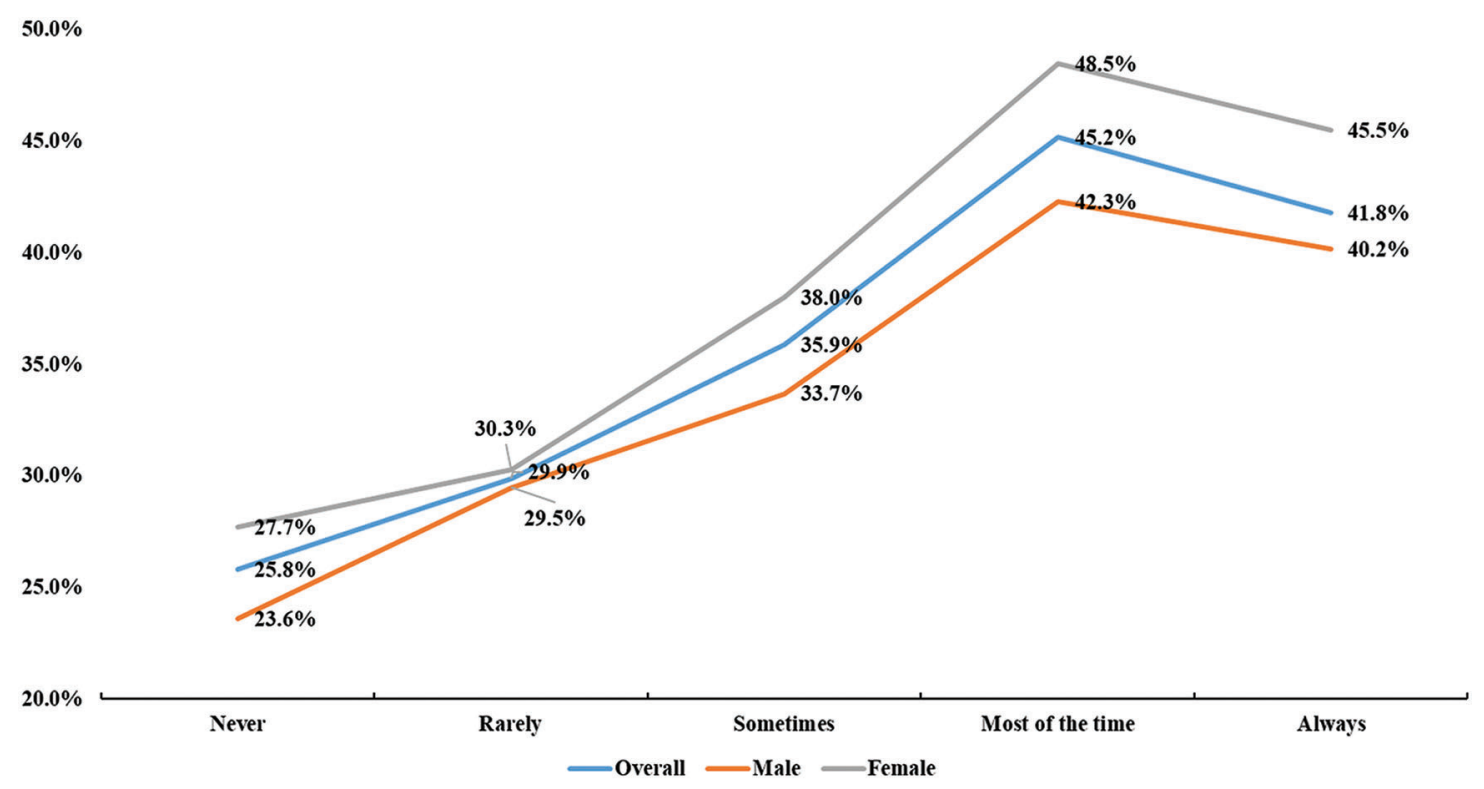

Figure 1: The prevalence of depressive symptoms by levels of food insecurity

Tab. 2 shows the results for the associations between FIS and depressive symptoms. Adolescents with any level of food insecurity were more likely to report having depressive symptoms than counterparts with lower levels of FIS, regardless of gender (rarely: $\mathrm{OR}=1.3,95 \% \mathrm{CI}$ : $1.2-1.4$; sometimes: $\mathrm{OR}=1.6,95 \% \mathrm{CI}$ : 1.5-1.8; most of the time: $\mathrm{OR}=2.1,95 \% \mathrm{CI}: 1.8-2.4$; always: $\mathrm{OR}=2.0,95 \% \mathrm{CI}: 1.6-2.4)$. From the observation of OR values, adolescents who reported food insecurity with most of the time have highest likelihood of having depressive symptoms for both male $(\mathrm{OR}=2.1,95 \% \mathrm{CI}$ : 1.7-2.6) and female $(\mathrm{OR}=2.1,95 \% \mathrm{CI}: 1.7-2.5)$.

Table 2: Associations between food insecurity and depressive symptoms (overall and by sex)

\begin{tabular}{|c|c|c|c|c|c|c|c|c|c|}
\hline \multirow[b]{3}{*}{ Rarely } & \multicolumn{3}{|c|}{ Overall } & \multicolumn{3}{|c|}{ Male } & \multicolumn{3}{|c|}{ Female } \\
\hline & \multirow{2}{*}{$\frac{\text { OR }}{1.3}$} & \multicolumn{2}{|c|}{$95 \%$ CI } & \multirow{2}{*}{$\frac{\text { OR }}{1.4}$} & \multicolumn{2}{|c|}{$95 \%$ CI } & \multirow{2}{*}{$\frac{\text { OR }}{1.2}$} & \multicolumn{2}{|c|}{$95 \%$ CI } \\
\hline & & 1.2 & 1.4 & & 1.2 & 1.7 & & 1.0 & 1.3 \\
\hline Sometimes & 1.6 & 1.5 & 1.8 & 1.6 & 1.4 & 1.8 & 1.7 & 1.4 & 1.9 \\
\hline Most of the time & 2.1 & 1.8 & 2.4 & 2.1 & 1.7 & 2.6 & 2.1 & 1.7 & 2.5 \\
\hline Always & 2.0 & 1.6 & 2.4 & 2.0 & 1.6 & 2.7 & 1.9 & 1.5 & 2.5 \\
\hline
\end{tabular}

Note: OR: odd ratio; CI: confidence interval; Reference group: reporting 'never'. 
Fig. 2 demonstrates the results with country-wise for the associations between FIS and depressive symptoms. The pooled estimate from a meta-analysis for all the samples was 1.60 (95\%CI: $1.52-1.69)$ with a small level of between-country heterogeneity $\left(I^{2}=12.7 \%\right)$. There was no significant heterogeneity between countries included in this study $(p=0.279)$.
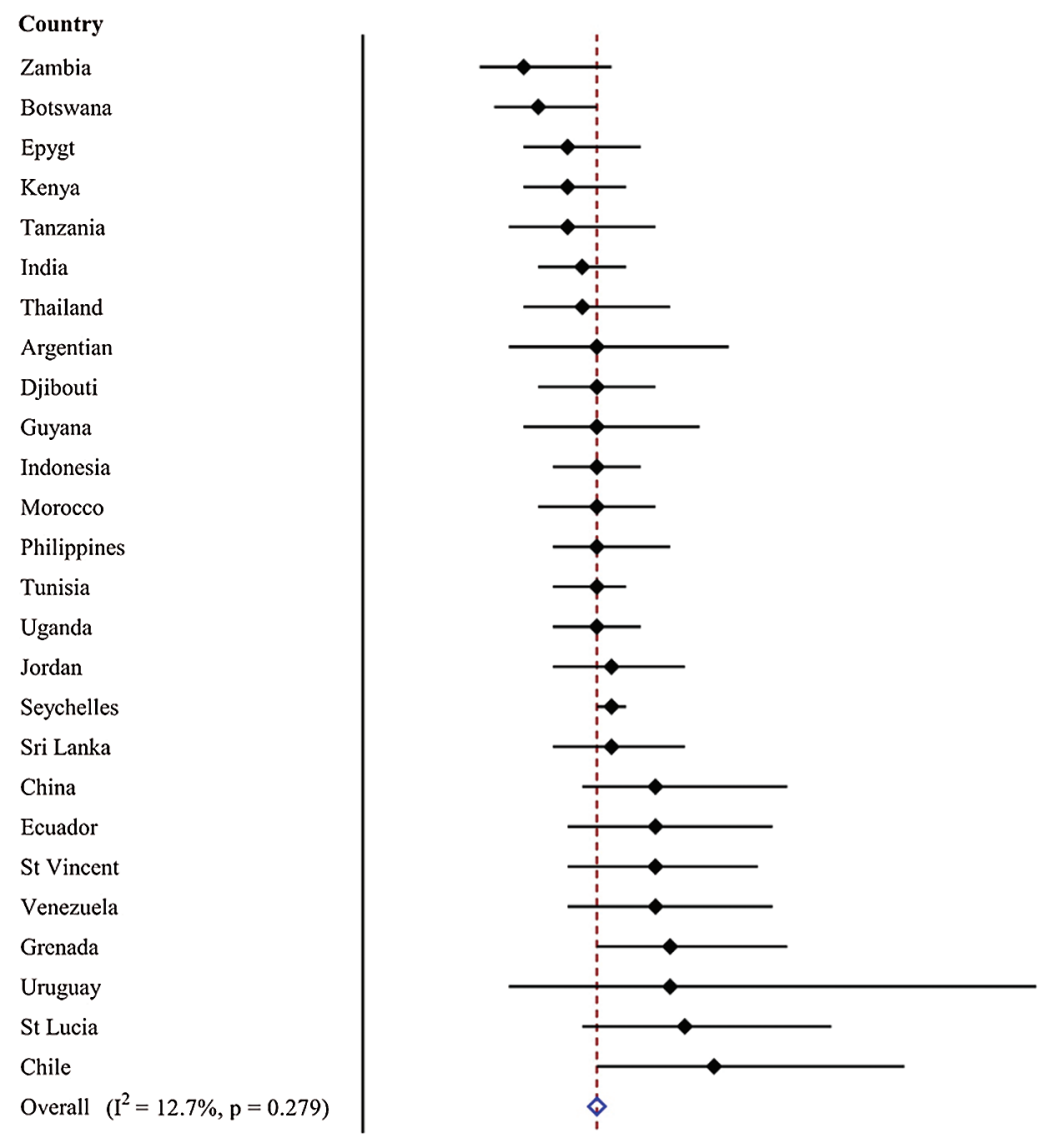

$\begin{array}{lll}\text { OR } & (95 \% \mathrm{CI}) & \text { Weight (\%) } \\ 1.10 & (0.80,1.70) & 3.05 \\ 1.20 & (0.90,1.60) & 4.77 \\ 1.40 & (1.10,1.90) & 3.77 \\ 1.40 & (1.10,1.80) & 4.77 \\ 1.40 & (1.00,2.00) & 2.51 \\ 1.50 & (1.20,1.80) & 6.17 \\ 1.50 & (1.10,2.10) & 2.51 \\ 1.60 & (1.00,2.50) & 1.17 \\ 1.60 & (1.20,2.00) & 3.77 \\ 1.60 & (1.10,2.30) & 1.79 \\ 1.60 & (1.30,1.90) & 6.17 \\ 1.60 & (1.20,2.00) & 3.77 \\ 1.60 & (1.30,2.10) & 3.77 \\ 1.60 & (1.30,1.80) & 8.24 \\ 1.60 & (1.30,1.90) & 6.17 \\ 1.70 & (1.30,2.20) & 3.05 \\ 1.70 & (1.60,1.80) & 22.70 \\ 1.70 & (1.30,2.20) & 3.05 \\ 2.00 & (1.50,2.90) & 1.33 \\ 2.00 & (1.40,2.80) & 1.33 \\ 2.00 & (1.40,2.70) & 1.53 \\ 2.00 & (1.40,2.80) & 1.33 \\ 2.10 & (1.60,2.90) & 1.53 \\ 2.10 & (1.00,4.60) & 0.21 \\ 2.20 & (1.50,3.20) & 0.91 \\ 2.40 & (1.60,3.70) & 0.60 \\ 1.60 & (1.52,1.69) & 100.00 \\ & & \end{array}$

Figure 2: Country-wise association between food insecurity (binary variable) and depressive symptoms estimated by multivariable logistic regression. Notes: $\mathrm{OR}=$ Odds ratio; $\mathrm{CI}=$ confidence interval; Models were adjusted for overall estimate was calculated by meta-analysis with random effects

\section{Discussion}

To our knowledge, our study is the first to examine the association between FIS and depressive symptoms in adolescents from LMICs and this study found that higher severity of FIS was significantly and positively associated with depressive symptoms in the studied population regardless of sex. The present study mainly found that the higher severity of FIS, the higher likelihood to be depressive except for the severity of FIS of being always. Moreover, there was a small country-wise inconsistency in the association between FIS and depressive symptoms. As previously published studies were mainly based on adult or other populations (e.g., pregnant women) [28,31,36-38], the current study could provide some would-be insightful and practical implications on policy of depressive symptoms in adolescents, especially for those who are from LMICs.

There has been much evidence to demonstrate the association between FIS and depressive symptoms in different populations, which is consistent with the present study. In young adults, there was a positive 
association between FIS and depressive symptoms [39,40]. A recent study based on Canadian adult populations indicated that household FIS was positively and significantly associated with depressive symptoms [41]. Two studies based on pregnant women also reported an association between FIS and depression [37,38]. In some population with specific diseases (e.g., like people with HIV), the association remains significant [42-44]. However, to our knowledge, little is known about the association in adolescents, especially from those living in LMICs. Across the literature, only one study consisting of adolescents and using parental reported household FIS, the authors found that FIS was associated with an increased risk of parent-assessed mental health status [45]. In a study with nationally representative samples from American suggested that children living with being food insecure were more likely to be diagnosed as depressive symptoms compared with those who live without food security [30]. Based on the mentioned-above evidence that can support our research finding that FIS was associated with depressive symptoms, our study adds more evidence to corroborate this association in adolescents. The abovementioned evidence suggests FIS, which particularly occurs in LMICs, are a risk factor of individuals' mental illness. Considering our study participants were from LMICs, the current study is of great importance to mental health promotion of young people with poor socioeconomic status.

Although our study assumed FIS as independent and found its positive association with depressive symptoms in adolescents from LMICs, some previous studies found that depressive symptoms might be a factor of FIS in adults [27,46,47]. Despite having such evidence, we cannot deny the adverse impacts of FIS on depressive symptoms. Of note, based on our study and previous research findings from crosssectional study, it is urgently required to explore the causal relationship between FIS and depressive symptoms, which can advance knowledge in the relevant research fields.

The possible reasons to support the significant association between FIS and depressive symptoms in this study are discussed below, which may help understand the underlying mechanism for the association. FIS would lead to worry and anxiety, which in turn makes people feel stressed and then developing as a factor of depressive symptoms [27]. In LMICs, it is highly likely that higher severity of FIS would make adolescent worry about their hungry and nutrition status, which contributes to their depressive symptoms. Another similar explanation is that FIS may make people feel deprivation and alienation [43]. From the perspective of social relationship, FIS also results in psycho-emotional burden by weakening social relationships because of low self-efficacy and a sense of helplessness [28,31]. Those factors may cause depressive symptoms. However, these assumed interpretations are mainly based on adults rather than adolescents, more studies are required to explore the age-specific mechanism for the association between FIS and depressive symptoms. Some evidence has demonstrated that young people with FIS are more likely to be exposed to emotional problems, like, which are contributors of depressive symptoms. Nevertheless, more insightful studies are required to answer why young people with FIS have higher odds for depressive symptoms.

The current study found a small percentage of heterogeneity resulted from country-wise comparison in the association between FIS and depressive symptoms $\left(I^{2}=12.7 \%\right)$. This research finding indicated that the association between FIS and depressive symptoms may not be varied by different countries. Possible reason for this small country-wise inconsistency of the association between FIS and depressive symptoms in our study involves study participants. In the current study, study participants were from LMICs, which implies that study participants were more likely to exposed to FIS and in turn tended to report depressive symptoms.

\subsection{Limitation and Strengths}

The research findings revealed here must be interpreted in light of some limitations. First, the study was a cross-sectional epidemiological survey, which fails to make casual inference for the association between FIS and depressive symptoms in adolescents. Future longitudinal studies are required to further confirm the association. Another limitation is the measures for FIS and depressive symptoms, which is subject to 
recall bias including measurement error and social desirability. Third, it is well-known that depressive symptoms of adolescents are also influenced by more other factors, but in our study, we failed to collect the relevant data. However, the strengths of this study include large-size sample and data from a multinational perspective. This research finding is useful in helping mental health promotion in a wider range of countries because of the higher generalization. Future research is urgently required to confirm the association between FI and depressive symptoms in adolescents based on casual-inference study. Moreover, to better establish the association, more accurate measures should be used.

\section{Conclusion}

This study offers evidence on the association between food insecurity and depressive symptoms in adolescents aged 12-15 yeas from low- and middle-income countries. Specifically, the high prevalence of food insecurity was reported in this study. And higher severity of food insecurity may increase the odds for depressive symptoms in adolescents. This significant association was not varied greatly by different countries. Based on the research findings, it is suggested that alleviating food insecurity of adolescents from low- and middle-income countries may be an effective approach to reduce health burden resulted from depression regardless of country-based situations.

Acknowledgement: The author appreciated the GSHS's public and available data.

Authors' Contribution: Study design, data analysis and manuscript draft were completed by the only author of this study.

Funding Statement: The author received no specific funding for this study.

Conflicts of Interest: The authors declare that they have no conflict of interest to report regarding the present study.

\section{References}

1. APA (1994). Diagnostic and statistical manual of mental disorders (DSM-IV-TR). Washington DC: American Psychiatric Association.

2. Li, J. Y., Li, J., Liang, J. H., Qian, S., Jia, R. X. et al. (2019). Depressive symptoms among children and adolescents in China: A systematic review and meta-analysis. Medical Science Monitor: International Medical Journal of Experimental \& Clinical Research, 25(22), 283-303.

3. Reddy, M. S. (2010). Depression: The disorder and the burden. Indian Journal of Psychological Medicine, 32(1), $1-2$. DOI 10.4103/0253-7176.70510.

4. Hankin, B. L. (2009). Development of sex differences in depressive and co-occurring anxious symptoms during adolescence: Descriptive trajectories and potential explanations in a multiwave prospective study. Journal of Clinical Child and Adolescent Psychology, 38(4), 460-472. DOI 10.1080/15374410902976288.

5. Adkins, D. E., Wang, V., Dupre, M. E., van den Oord, E. J., Elder, G. H. et al. (2009). Structure and stress: Trajectories of depressive symptoms across adolescence and young adulthood. Social Forces, 88(1), 31-60. DOI 10.1353/sof.0.0238.

6. Ahmed, S. P., Bittencourt-Hewitt, A., Sebastian, C. L. (2015). Neurocognitive bases of emotion regulation development in adolescence. Development of Cognitive Neuroscience, 15(3), 11-25. DOI 10.1016/j.den.2015.07.006.

7. Olufowote, R. A. D., Soloski, K. L., Gonzalez-Casteneda, N., Hayes, N. D. (2020). An accelerated latent class growth curve analysis of adolescent bonds and trajectories of depressive symptoms. Journal of Child \& Family Studies, 29(3), 34-49.

8. Pileggi, L. A., Storey, S., Malcolm-Smith, S. (2020). Depressive symptoms disrupt leftward cradling. Journal of Child \& Adolescent Mental Health, 32(1), 35-43. DOI 10.2989/17280583.2020.1765786. 
9. Carrellas, N. W., Biederman, J., Uchida, M. (2017). How prevalent and morbid are subthreshold manifestations of major depression in adolescents? A literature review. Journal of Affective Disorders, 210(32), 166-173. DOI 10.1016/j.jad.2016.12.037.

10. Axelsdóttir, B., Biedilæ, S., Sagatun, Å., Nordheim, L. V., Larun, L. (2020). Review: Exercise for depression in children and adolescents-A systematic review and meta-analysis. Child \& Adolescent Mental Health.

11. Kelly, R. H., Russo, J., Holt, V. L., Danielsen, B. H., Zatzick, D. F. et al. (2002). Psychiatric and substance use disorders as risk factors for low birth weight and preterm delivery. Obstetrics \& Gynecology, 100(2), 297-304.

12. Wagner, S., Müller, C., Helmreich, I., Huss, M., Tadić, A. (2015). A meta-analysis of cognitive functions in children and adolescents with major depressive disorder. European Child \& Adolescent Psychiatry, 24(1), 519. DOI 10.1007/s00787-014-0559-2.

13. Karadag, M. (2020). Evaluation of depression and anxiety levels in school dropout adolescents. Journal of Clinical Psychiatry, 23(3), 47-59.

14. Goldstein, A. L., Faulkner, B., Wekerle, C. (2013). The relationship among internal resilience, smoking, alcohol use, and depression symptoms in emerging adults transitioning out of child welfare. Child Abuse \& Neglect, 37(1), 22-32. DOI 10.1016/j.chiabu.2012.08.007.

15. Liu, J., Liu, C. X., Wu, T., Liu, B. P., Jia, C. X. et al. (2019). Prolonged mobile phone use is associated with depressive symptoms in Chinese adolescents. Journal of Affective Disorders, 259, 128-134. DOI 10.1016/j. jad.2019.08.017.

16. Sampasa-Kanyinga, H., Colman, I., Goldfield, G. S., Janssen, I., Wang, J. et al. (2020). Combinations of physical activity, sedentary time, and sleep duration and their associations with depressive symptoms and other mental health problems in children and adolescents: A systematic review. International Journal of Behavioral Nutrition and Physical Activity, 17(1), 1-16. DOI 10.1186/s12966-020-00976-x.

17. Kandola, A., Lewis, G., Osborn, D. P., Stubbs, B., Hayes, J. F. (2020). Depressive symptoms and objectively measured physical activity and sedentary behaviour throughout adolescence: A prospective cohort study. Lancet Psychiatry, 7(3), 262-271. DOI 10.1016/S2215-0366(20)30034-1.

18. Chi, X., Huang, L., Wang, J., Zhang, P. (2020). The prevalence and socio-demographic correlates of depressive symptoms in early adolescents in China: Differences in only child and non-only child groups. International Journal of Environmental Research and Public Health, 17(2), 438. DOI 10.3390/ijerph17020438.

19. Vancampfort, D., Stubbs, B., Firth, J., van Damme, T., Koyanagi, A. (2018). Sedentary behavior and depressive symptoms among 67,077 adolescents aged 12-15 years from 30 low- and middle-income countries. International Journal of Behavioral Nutrition and Physical Activity, 15(1), 73. DOI 10.1186/s12966-018-0708-y.

20. Gundersen, C., Ziliak, J. (2015). Food insecurity and health outcomes. Health Affairs, 34(11), 1830-1839. DOI 10.1377/hlthaff.2015.0645.

21. Jebena, M. G., Lindstrom, D., Lachat, C., Belachew, T., Kolsteren, P. (2017). The effect of food insecurity on health status of adolescents in Ethiopia: Longitudinal study. BMC Public Health, 17(1), 1-12. DOI 10.1186/ s12889-017-4406-5.

22. Hatem, C., Lee, C. Y., Zhao, X., Reesor-Oyer, L., Lopez, T. et al. (2020). Food insecurity and housing instability during early childhood as predictors of adolescent mental health. Journal of Family Psychology, 34(6), 721-730. DOI $10.1037 /$ fam0000651.

23. Howard, L. L. (2011). Does food insecurity at home affect non-cognitive performance at school? A longitudinal analysis of elementary student classroom behavior. Economics of Education Review, 30(1), 157-176. DOI 10.1016/j.econedurev.2010.08.003.

24. Koyanagi, A., Stubbs, B., Oh, H., Veronese, N., Smith, L. et al. (2019). Food insecurity (hunger) and suicide attempts among 179,771 adolescents attending school from 9 high-income, 31 middle-income, and 4 lowincome countries: A cross-sectional study. Journal of Affective Disorders, 248, 91-98. DOI 10.1016/j. jad.2019.01.033.

25. Lee, Y. S., Kim, T. H. (2019). Household food insecurity and breakfast skipping: Their association with depressive symptoms. Psychiatry Research, 271, 83-88. DOI 10.1016/j.psychres.2018.11.031. 
26. Kolovos, S., Zavala, G. A., Leijen, A. S., Melgar-Quiñonez, H., van Tulder, M. (2020). Household food insecurity is associated with depressive symptoms: Results from a Mexican population-based survey. Food Security, 12(2), 407- 416. DOI 10.1007/s12571-020-01014-1.

27. Maynard, M., Andrade, L., Packull-McCormick, S., Perlman, C. M., Leos-Toro, C. et al. (2018). Food insecurity and mental health among females in high-income countries. International Journal of Environmental Research and Public Health, 15(7), 1424. DOI 10.3390/ijerph15071424.

28. Pourmotabbed, A., Moradi, S., Babaei, A., Ghavami, A., Mohammadi, H. et al. (2020). Food insecurity and mental health: A systematic review and meta-analysis. Public Health Nutrition, 23(10), 1778-1790. DOI 10.1017/ S136898001900435X.

29. Thomas, M. M., Miller, D. P., Morrissey, T. W. (2019). Food insecurity and child health. Pediatrics, 144(4), e20190397. DOI 10.1542/peds.2019-0397.

30. Bruening, M., Dinour, L. M., Chavez, J. B. R. (2017). Food insecurity and emotional health in the USA: A systematic narrative review of longitudinal research. Public Health Nutrition, 20(17), 3200-3208. DOI $10.1017 / \mathrm{S} 1368980017002221$.

31. Felez-Nobrega, M., Haro, J. M., Vancampfort, D., Koyanagi, A. (2020). Sex difference in the association between physical activity and suicide attempts among adolescents from 48 countries: A global perspective. Journal of Affective Disorders, 266(3), 311-318. DOI 10.1016/j.jad.2020.01.147.

32. Koyanagi, A., Oh, H., Carvalho, A. F., Smith, L., Haro, J. M. et al. (2019). Bullying victimization and suicide attempt among adolescents aged 12-15 years from 48 countries. Journal of the American Academy of Child and Adolescent Psychiatry, 58(9), 907-918. DOI 10.1016/j.jaac.2018.10.018.

33. Gu, J., Chen, S. T. (2020). Association between active travel to school and depressive symptoms among early adolescents. Children, 7(5), 41. DOI 10.3390/children7050041.

34. Chen, S. T., Guo, T., Yu, Q., Stubbs, B., Clark, C. et al. (2021). Active school travel is associated with fewer suicide attempts among adolescents from low-and middle-income countries. International Journal of Clinical and Health Psychology, 21(1), 100202. DOI 10.1016/j.ijchp.2020.11.001.

35. Raab, R., Michel, S., Günther, J., Hoffmann, J., Stecher, L. et al. (2021). Associations between lifestyle interventions during pregnancy and childhood weight and growth: A systematic review and meta-analysis. International Journal of Behavioral Nutrition and Physical Activity, 18(1), 1-14. DOI 10.1186/s12966-02001075-7.

36. Abrahams, Z., Lund, C., Field, S., Honikman, S. (2018). Factors associated with household food insecurity and depression in pregnant South African women from a low socio-economic setting: A cross-sectional study. Social Psychiatry and Psychiatric Epidemiology, 53(4), 363-372. DOI 10.1007/s00127-018-1497-y.

37. Hromi-Fiedler, A., Bermúdez-Millán, A., Segura-Pérez, S., Pérez-Escamilla, R. (2011). Household food insecurity is associated with depressive symptoms among low-income pregnant Latinas. Maternal \& Child Nutrition, 7(4), 421-430. DOI 10.1111/j.1740-8709.2010.00266.x.

38. Pryor, L., Lioret, S., van der Waerden, J., Fombonne, É., Falissard, B. et al. (2016). Food insecurity and mental health problems among a community sample of young adults. Social Psychiatry and Psychiatric Epidemiology, 51(8), 1073-1081. DOI 10.1007/s00127-016-1249-9.

39. Darling, K. E., Fahrenkamp, A. J., Wilson, S. M., D’Auria, A. L., Sato, A. F. (2017). Physical and mental health outcomes associated with prior food insecurity among young adults. Journal of Health Psychology, 22(5), 572581. DOI 10.1177/1359105315609087.

40. Shafiee, M., Vatanparast, H., Janzen, B., Serahati, S., Keshavarz, P. et al. (2021). Household food insecurity is associated with depressive symptoms in the Canadian adult population. Journal of Affective Disorders, 279, 563-571. DOI 10.1016/j.jad.2020.10.057.

41. Palar, K., Frongillo, E. A., Escobar, J., Sheira, L. A., Wilson, T. E. et al. (2018). Food insecurity, internalized stigma, and depressive symptoms among women living with HIV in the United States. AIDS and Behavior, 22(12), 3869-3878. DOI 10.1007/s10461-018-2164-8. 
42. Palar, K., Kushel, M., Frongillo, E. A., Riley, E. D., Grede, N. et al. (2015). Food insecurity is longitudinally associated with depressive symptoms among homeless and Marginally-housed individuals living with HIV. AIDS and Behavior, 19(8), 1527-1534. DOI 10.1007/s10461-014-0922-9.

43. Tesfaye, M., Kaestel, P., Olsen, M. F., Girma, T., Yilma, D. et al. (2016). Food insecurity, mental health and quality of life among people living with HIV commencing antiretroviral treatment in Ethiopia: A cross-sectional study. Health and Quality of Life Outcomes, 14(1), 1-8. DOI 10.1186/s12955-016-0440-8.

44. Poole-Di Salvo, E., Silver, E. J., Stein, R. E. (2016). Household food insecurity and mental health problems among adolescents: What do parents report? Academic Pediatrics, 16(1), 90-96. DOI 10.1016/j.acap.2015.08.005.

45. Ahuja, M., Sathiyaseelan, T., Wani, R. J., Fernandopulle, P. (2020). Obesity, food insecurity, and depression among females. Archives of Public Health, 78(1), 1-6. DOI 10.1186/s13690-020-00463-6.

46. Isaura, E. R., Chen, Y. C., Adi, A. C., Fan, H. Y., Li, C. Y. et al. (2019). Association between depressive symptoms and food insecurity among Indonesian adults: Results from the 2007-2014 Indonesia family life survey. Nutrients, 11(12), 3026. DOI 10.3390/nu11123026.

47. Althoff, R. R., Ametti, M., Bertmann, F. (2016). The role of food insecurity in developmental psychopathology. Preventive Medicine, 92, 106-109. DOI 10.1016/j.ypmed.2016.08.012. 